\title{
High-harmonic generation at the nanoscale boosted by bound states in the continuum
}

\author{
Luca Carletti $\odot,{ }^{1, *}$ Sergey S. Kruk, ${ }^{2}$ Andrey A. Bogdanov, ${ }^{3}$ Costantino De Angelis, ${ }^{4}$ and Yuri Kivshar ${ }^{2,3}$ \\ ${ }^{1}$ Department of Information Engineering, University of Padova, Padova 35131, Italy \\ ${ }^{2}$ Nonlinear Physics Centre, Australian National University, Canberra ACT 2601, Australia \\ ${ }^{3}$ ITMO University, St. Petersburg 197101, Russia \\ ${ }^{4}$ Department of Information Engineering, University of Brescia and National Institute of Optics (INO-CNR), Brescia 25123, Italy
}

(Received 24 May 2019; revised manuscript received 8 August 2019; published 13 September 2019)

\begin{abstract}
Recent progress in nanoscale optics is driven by the physics of electric and magnetic resonances supported by high-index dielectric nanoparticles. Here, we exploit optical bound states in the continuum in a subwavelength particle enhanced by an engineered substrate undergoing an epsilon-near-zero transition from an insulator to a conductor, and uncover how to boost dramatically high-order parametric nonlinear effects. Our strategy makes feasible an observation of a variety of multistep cascaded and multifrequency nonlinear effects in an individual subwavelength resonator. This would expand substantially the range of applications of Mieresonant dielectric metaphotonics for highly efficient subwavelength optical circuitry, nonlinear metadevices, ultrasensitive hyperspectral sensing, and quantum nanophotonics.
\end{abstract}

DOI: 10.1103/PhysRevResearch.1.023016

\section{INTRODUCTION}

Frequency conversion is one of the major applications of nonlinear optics, and it relies on light-matter interaction to produce an efficient exchange of energy from one frequency to another [1]. When the size of a photonic structure becomes less than the incoming wavelength, the phase-matching engineering is no longer applicable to improve the conversion efficiency. Thus, new concepts have to be developed to enhance nonlinear optical phenomena at the nanoscale. One strategy is to employ optical cavities with resonances both at the fundamental and harmonic frequencies [2]. However, this approach has yet to lead to the demonstration of a large efficiency due to mode-symmetry selection rules [3-5]. A substantial progress in nonlinear optics at the nanoscale was achieved with the use of plasmonic structures [6]. However, the efficiency of nonlinear effects in such structures remains extremely low due to high losses and heating limiting the maximum powers. Optical nonlinear effects could be enhanced further in hybrid structures and novel materials [7-10], but different approaches are required.

A recently emerged and rapidly developing prospective platform for nonlinear photonics is a nanostructure made of high-refractive-index materials $[11,12]$. The efficiency of nonlinear effects in such systems can be enhanced because of low heating allowing high pump powers and confinement of the electromagnetic fields inside the structures. This approach is promising to enable more efficient nonlinear metadevices at the nanoscale [13].

\section{*Corresponding author: luca.carletti@unipd.it}

Published by the American Physical Society under the terms of the Creative Commons Attribution 4.0 International license. Further distribution of this work must maintain attribution to the author(s) and the published article's title, journal citation, and DOI.
The first demonstration of third-harmonic generation (THG) in silicon nanoparticles driven by a magnetic dipole resonance achieved a conversion efficiency enhancement by two orders of magnitude with respect to an unstructured silicon slab [14]. Recently, anapole resonance in a silicon nanodisk on a gold substrate enabled THG with $10^{-4}$ conversion efficiency [15]. Degenerate wave mixing driven by third-order nonlinearities in isolated germanium and silicon nanodisks has also been reported recently [16,17]. Second-order nonlinear effects in Mie resonators have been considered as well in III-V semiconductors, in perovskites, and in metal-oxide nanoparticles [18-24]. Very recently, multifrequency effects have been demonstrated in resonant dielectric metasurfaces [23,25]. All those effects relied on the interplay between Mie resonances and collective Fano-type resonances of two-dimensional lattices. In individual isolated nanoparticles, however, the overall efficiency of nonlinear processes has remained relatively low [26]. As a result, multistep cascaded and multifrequency nonlinear processes, such as those shown schematically in Figs. 1(a) and 1(b), are still to be demonstrated.

Importantly, as suggested recently [27,28], in addition to magnetic dipole Mie-type and anapolar resonances, individual high-index dielectric nanoparticles can support nontrivial optical modes in the subwavelength regime which originate from the physics of bound states in the continuum (BIC) [29]. It was then predicted that such novel nonradiating optical states can be used to increase the efficiencies of second-harmonic generation by orders of magnitude [30-32], thus offering a paradigm shift for nonlinear nanoscale optics.

In this paper, we combine the concept of BIC with smart engineering of the substrate that undergoes a transition from an insulator to a conductor via an epsilon-near-zero (ENZ) regime [33,34] and reveal how to boost the efficiency of multifrequency [25] and multistep [35,36] nonlinear processes at the nanoscale. We would like to stress that the function of the ENZ material is solely as a reflector with limited 

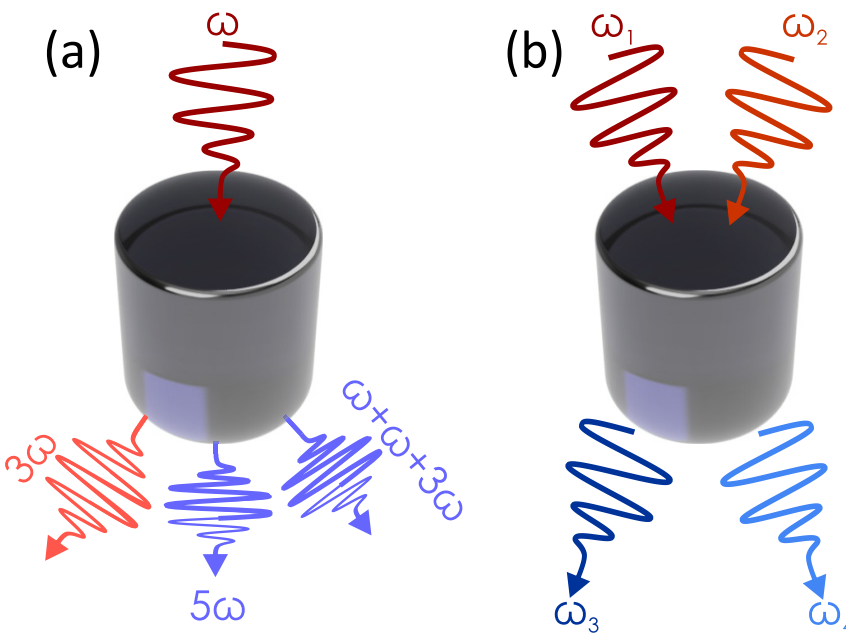

FIG. 1. Conceptual presentation of direct, cascaded, and multifrequency nonlinear parametric effects at the nanoscale. (a) Photons of a pump beam at the frequency $\omega$ are converted to photons at $3 \omega$, via third-harmonic generation, and $5 \omega$, via fifth-harmonic generation, and two-step cascading. (b) Photons at two frequencies $\omega_{1}$ and $\omega_{2}$ are mixed by degenerate four-wave mixing to generate photons at the frequencies $\omega_{3}=2 \omega_{1}+\omega_{2}$ and $\omega_{4}=2 \omega_{2}+\omega_{1}$.

absorption loss and it is not considered as nonlinear material. Our results suggest how to expand substantially the range of applications of resonant dielectric nanophotonics for highly efficient nonlinear metadevices and quantum nanophotonics.

\section{MODEL AND APPROACH}

An example of the structures that we consider is represented in Fig. 2(a). It consists of a dielectric nanodisk resonator of radius $r$ and height $t$ placed on an engineered multilayered substrate made of a layer of epsilon-near-zero (ENZ) material sandwiched between a thin layer of an insulator and a dielectric substrate. In our specific case, we select a silicon nanoparticle placed on a buffer low-index dielectric layer (such as $\mathrm{SiO}_{2}$ or $\mathrm{CaF}_{2}$ ) of thickness $h$, and a 300-nm-thin layer of indium tin oxide (ITO). The complex permittivity functions of silicon and ITO are taken from experimental data $[33,37]$. The refractive index of the buffer layer is assumed to be 1.44 and dispersionless.

We use the eigenmode analysis for the characterization and optimization of the resonant modes of the system. The eigenmodes of the photonic structure are calculated with the modal solver implemented in COMSOL MULTIPHYSICS. We focus on the study of rotationally symmetric resonant modes, for which electromagnetic field does not depend on azimuthal angle $\phi$ [Fig. 2(a)]. The calculations are performed for the fixed values of material permittivity $\varepsilon_{\mathrm{Si}}=12.052$, $\varepsilon_{\text {ITO }}=-2.668+i 0.711$ chosen at wavelength $1600 \mathrm{~nm}$. For the buffer layer we take, as reference, the permittivity of $\mathrm{SiO}_{2}$ at a wavelength of $1600 \mathrm{~nm}, \varepsilon_{\mathrm{SiO}_{2}}=2.084$. Figure 2(b) shows the resonant modes of disks with $h=300 \mathrm{~nm}$ as a function of the normalized geometrical parameters defined as the disk aspect ratio $(\mathrm{AR}=r / t)$ and the size parameter $(\mathrm{SP}=r / \lambda)$. The quality factor ( $Q$ factor) is shown in the color scale. In certain range of parameters we observe two avoid crossings accompanied by appearance of quasi-BICs at (SP; AR) of about $(0.276 ; 0.73)$ and $(0.303 ; 0.57)$. Hereafter, we consider the quasi-BIC at the smallest size parameter [see red-shaded area in Fig. 2(b)] as it is more attractive for compact frequency conversion devices. The peak value of the $Q$ factor of 310 is reached for the disks of a radius $441 \mathrm{~nm}$ and a height $605 \mathrm{~nm}$.

It is worth to mention that a true $\mathrm{BIC}$ with an infinite- $Q$ factor is a mathematical abstraction as it requires either infinite size of the structure or zero (or infinite) permittivity. However, a physical mechanism that can originate BICs is the destructive interference between two modes with a similar far-field radiation pattern in the strong coupling regime [38]. Likewise, in isolated photonic resonators the radiating tails of two leaky modes can cancel almost completely the far field of each other in a strong coupling regime via destructive interference. As a consequence, one of these modes exhibits a finite but high- $Q$ factor $[27,28]$. The same physics underlying appearance of infinitely high- $Q$ states in periodic structures (BIC) and high- $Q$ states in a single dielectric resonators allows for
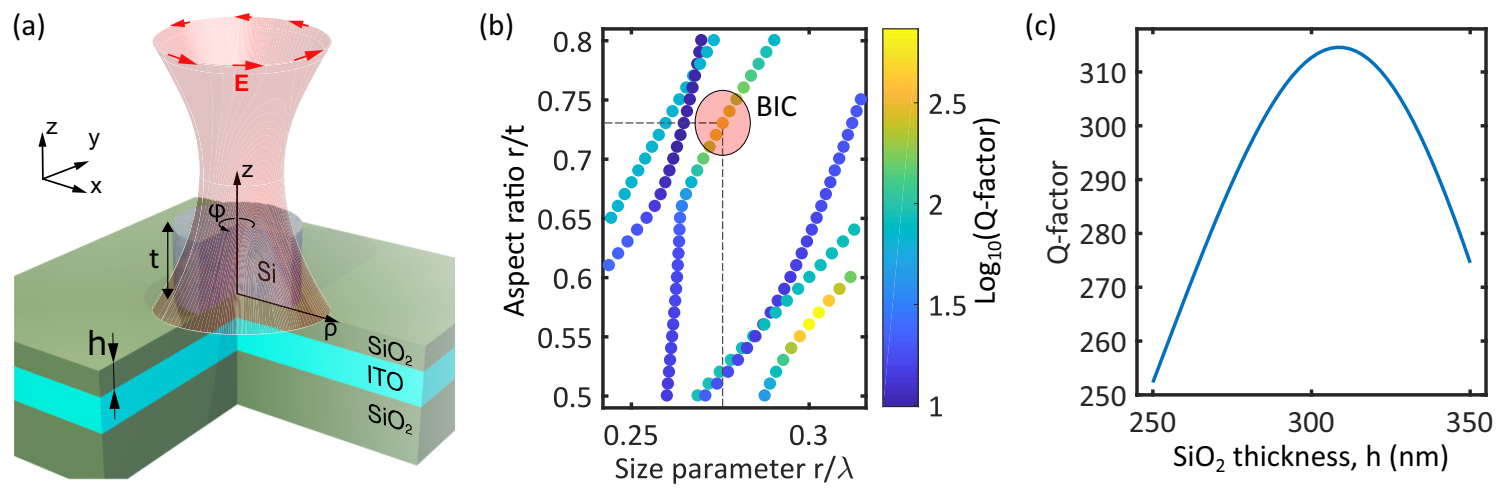

FIG. 2. Exploration of bound states in the continuum. (a) Schematic of the structure. The Si disk resonator is placed on top of a multilayer substrate. This is composed of a $\mathrm{SiO}_{2}$ layer of thickness $h$, a 300-nm layer of ITO, and another semi-infinite layer of $\mathrm{SiO}_{2}$. The $\mathrm{SiO}_{2}$ could be replaced by other materials exhibiting low refractive index and no absorption at the wavelengths of interests such as $\mathrm{CaF}_{2}, \mathrm{Al}_{2} \mathrm{O}_{3}, \mathrm{or}_{\mathrm{MgF}}$. Cylindrical coordinate system $(\rho, \phi, z)$ is depicted in the drawing. The pump beam is azimuthally polarized. (b) Dispersion relation of radially symmetric resonant modes. The color of each point represents the $Q$ factor in the logarithmic scale. Dashed lines indicate the parameters of the quasi-BIC. (c) Maximum $Q$ factor of the quasi-BIC as a function of the thickness $h$. 


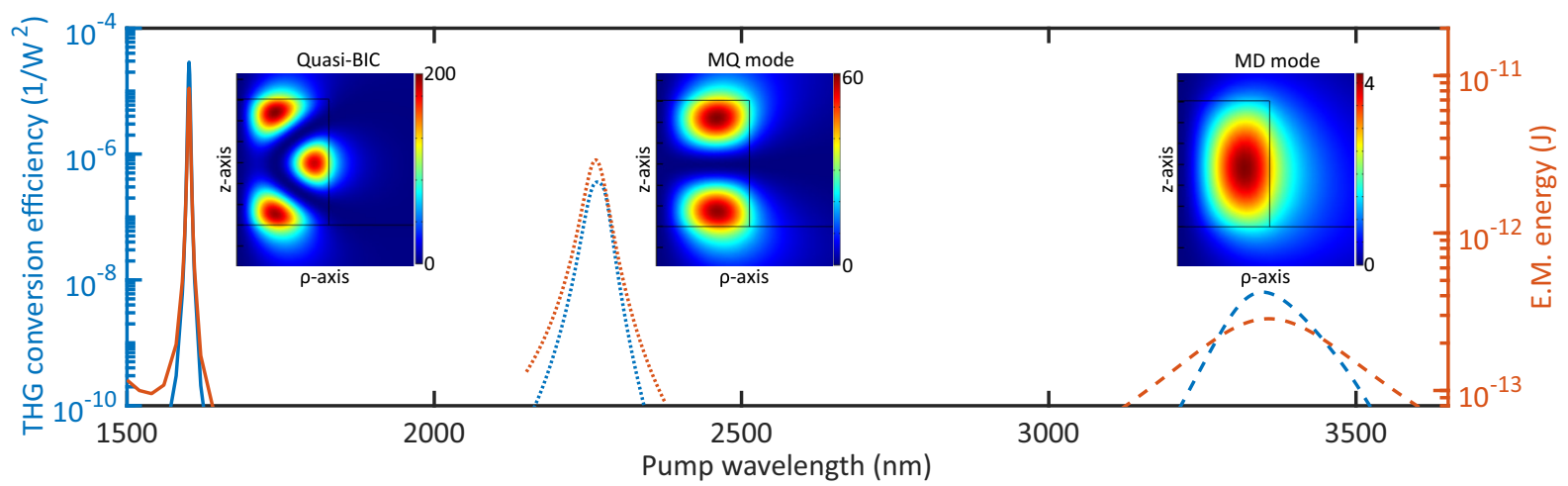

FIG. 3. Conversion efficiency for THG as a function of the pump wavelength close to quasi-BIC, MQ mode, and MD mode. Electromagnetic energy in the disk at the pump wavelength is shown on the right $y$ axis. Insets show the electric field enhancement in the $(\rho, z)$ plane at an incident wavelength of $1601 \mathrm{~nm}$ (quasi-BIC), $2260 \mathrm{~nm}$ (MQ mode), and $3360 \mathrm{~nm}$ (MD mode), respectively.

calling the latter quasi-BIC. This mechanism, that has been already predicted theoretically and observed experimentally [27-29], is responsible for the formation of the states with relatively high- $Q$ factor in our structure. Indeed, by continuously varying the geometrical parameters we control the coupling between the two modes reaching the strong coupling regime when one of the modes turns into a quasi-BIC [27]. Interestingly, the $Q$ factor can be increased by tuning the thickness of spacer made of the low-refractive index material. In Fig. 2(c) we show the mode $Q$ factor as a function of the thickness $h$ of the spacer. As we expected, there is an optimal thickness $(h=310 \mathrm{~nm})$ that maximizes the $Q$ factor that achieves a value of 315 . This value is very high for isolated high-index dielectric resonators at optical frequencies. Values of about 100 have been previously predicted without the substrate [30]. Such an increase of the $Q$ factor can be explained by destructive interference between the out-of-plane magnetic multipoles that contribute to the quasi-BIC resonance and their image in the substrate $[39,40]$. The low refractive index buffer layer between the resonator and ITO provides the correct phase difference between the fields radiated by the cavity and its mirror image to suppress radiation leakages. Furthermore, the use of an ENZ material instead of a metal, as in Refs. [39,40], provides the advantage to feature very high reflectivity with smaller absorption loss. Thus, as demonstrated here, the use of a smart substrate not only improves the $Q$ factor of the resonant mode, but also renders these photonic systems more realistic for fabrication. Moreover, this optimization procedure is rather general, and it could be used to design resonances at a different wavelength and using different materials or resonator geometries.

\section{NONLINEAR EFFECTS}

To study the nonlinear response of our structure, we use three-dimensional electromagnetic simulations implemented with the finite-element method in COMSOL MULTIPHYSICS. The nonlinear optical scattering of the nanoresonator is calculated in the undepleted pump approximation, and it is obtained by employing a two-step procedure. In the first step, the linear problem at the pump frequency is solved. The pump beam considered here is azimuthally polarized and it is focused in a diffraction-limited spot with a diameter equal to $1.22 \times \lambda / \mathrm{NA}$, where $\mathrm{NA}$ is a numerical aperture of 0.85 . Such a polarized beam provides the most efficient coupling to the rotationally symmetric electromagnetic modes. Since both the geometry and the electromagnetic fields exhibit axial symmetry, we adopt a cylindrical coordinate system, as shown in Fig. 2(a), to reduce the computational load. In the second step, we solve the problem describing the generation of the new frequency components. The source term in this step is introduced as the currents induced by the incident beam via nonlinear susceptibility. As previously observed, the high- $Q$ mode that we are investigating here is best excited with a focused azimuthally polarized beam [see Fig. 1(a)] [30]. Thus, we exploit the axial symmetry of the problem and use a cylindrical coordinate system, as shown in Fig. 2(a), to reduce the computational load.

\section{A. Third-harmonic generation}

Our structure is rather general, and it may be employed for the enhancement of many nonlinear effects at the nanoscale $[6,26]$. The first example that we use to outline the potential of our approach is THG. The nonlinear polarization at $3 \omega$ is given by $\mathbf{P}^{3 \omega}=3 \varepsilon_{0} \chi^{(3)}(\mathbf{E} \cdot \mathbf{E}) \mathbf{E}^{\omega}$, where $\varepsilon_{0}$ is the vacuum permittivity, $\chi^{(3)}=2.45 \times 10^{-19}(\mathrm{~m} / \mathrm{V})^{2}$ is the third-order nonlinear susceptibility, and $\mathbf{E}^{\omega}$ is the electric field at the pump frequency $\omega$. Figure 3 shows the spectrum of the normalized THG conversion efficiency $\zeta=P_{\mathrm{TH}} / P_{\mathrm{FF}}^{3}$, where $P_{\mathrm{TH}}$ is the scattered power at the third harmonic (TH) and $P_{\mathrm{FF}}$ is the incident power at the fundamental frequency $(\mathrm{FF})$. At variance with other THG studies $[14,15]$, here we consider $\zeta$ instead of the ratio $P_{\mathrm{TH}} / P_{\mathrm{FF}}$ because $\zeta$ does not depend on the pump power. The electromagnetic (EM) energy in the nanodisk as a function of the wavelength is reported on the right axis of the same plot. At the quasi-BIC wavelength of $1600 \mathrm{~nm}, \zeta$ (solid blue line) is enhanced by more than five orders of magnitude in comparison with wavelengths detuned from the quasi-BIC. It can also be seen from Fig. 3 that the cavity energy at the pump wavelength (contiguous orange line) exhibits a narrow peak at the quasi-BIC wavelength. This illustrates clearly the huge enhancement of the THG efficiency when the pump wavelength is tuned to the quasi-BIC. The peak THG conversion efficiency reaches the value of about $3 \times 10^{-5} \mathrm{~W}^{-2}$. 
TABLE I. Enhancement of the THG with resonant Si nanodisks. The columns from left to right report the reference, TH average power, FF average power, temporal duration of the pump pulse, repetition rate of the pump pulses, conversion efficiency $\eta$, and THG nonlinear coefficient $\zeta$

\begin{tabular}{lcccccc}
\hline \hline Reference & \multirow{2}{*}{ TH average power $(\mathrm{nW})$} & FF average power $(\mathrm{mW})$ & Pump pulse duration (fs) & Rep. rate $(\mathrm{MHz})$ & $\eta$ & $\zeta\left(1 / \mathrm{W}^{2}\right)$ \\
\hline$[14]$ & 4 & 34 & 200 & 80 & $1.2 \times 10^{-7}$ & $2.6 \times 10^{-14}$ \\
{$[15]$} & 16.8 & 0.14 & 100 & 80 & $1.2 \times 10^{-4}$ & $3.9 \times 10^{-7}$ \\
This work & $14.8 \times 10^{3}$ & 0.32 & 100 & 80 & $4.6 \times 10^{-2}$ & $2.9 \times 10^{-5}$ \\
\hline \hline
\end{tabular}

To compare the efficiency of the THG process predicted in this work with the experimental data from Refs. $[14,15]$ we use the following figure of merit (FOM) $\zeta=P_{\mathrm{TH}}^{p} /\left(P_{\mathrm{FF}}^{p}\right)^{3}$, where $P_{\mathrm{TH}}^{p}$ is the scattered peak power at the third harmonic (TH) and $P_{\mathrm{FF}}^{p}$ is the incident peak power at the fundamental frequency (FF). This FOM is thus independent of the pump power used for THG, allowing us to illustrate a more fair comparison. However, for practical applications it is also interesting to know the conversion efficiency that is defined as $\eta=P_{\mathrm{TH}}^{a} / P_{\mathrm{FF}}^{a}$, where $P_{\mathrm{TH}}^{a}$ and $P_{\mathrm{FF}}^{a}$ are the $\mathrm{TH}$ and FF average powers. It is clear from this definition that, being the THG signal intensity dependent on the FF power, the value of $\eta$ scales quadratically with the pump power. Moreover, since the studied optical nonlinearities manifest only for very high-field intensities, the value of $\eta$ strongly depends on the actual peak power of the FF beam. Thus, for our analysis, we used the laser pulse duration $T$ and repetition rate $R$ to link the peak and average powers as $P^{p}=P^{a} \times T \times R$. Finally, to compare our theoretical results to the experimental demonstrations, we calculated average powers of $\mathrm{TH}$ and FF signals from the FF peak power used in the simulation to operate at an intensity of $1 \mathrm{GW} / \mathrm{cm}^{2}(40 \mathrm{~W})$ and the generated $\mathrm{TH}$ power $(1.85 \mathrm{~mW})$. The values that we obtain with this analysis are reported in Table I, and they show that the $\mathrm{TH}$ conversion efficiency that we predict in this work is $10^{9}$ times higher than that in Ref. [14], and two orders of magnitudes higher than record-high THG in Ref. [15].

The asset of our approach is the use of a mode with an enhanced $Q$ factor. To gain deeper insight into the impact of the mode $Q$ factor on the THG conversion efficiency, we compare these results with the efficiency obtained in the same disk but in the vicinity of the magnetic quadrupole (MQ) and magnetic dipole (MD) modes that are excited at longer wavelengths than quasi-BIC. Comparison between these modes is justified because all of them have the same symmetry and, therefore, they can be excited with the same azimuthally polarized Gaussian beam. In the numerical simulations, we have considered a dispersionless material with constant permittivity of $\varepsilon=2.084$ for the buffer layer. The THG conversion efficiency shown in Fig. 3 driven by MQ (dotted blue line) and MD (dashed blue line) resonances are, respectively, three and two orders of magnitude smaller than the BIC-driven THG. This is explained by lower- $Q$ factors and, in turn, lower-field enhancement at MD and MQ resonances in comparison with quasi-BIC $\left(Q_{\mathrm{MD}} \approx 40\right.$ and $\left.Q_{\mathrm{MQ}} \approx 10\right)$.

\section{B. Four-wave mixing}

The generation of light at new frequencies exploiting third-order optical nonlinearities can be strongly enhanced in functionalities by using four-wave mixing (FWM). However, due to the complexity of this phenomenon that involves multiple frequencies, the conversion efficiency in FWM is limited by the hurdle of realizing spectral and spatial matching between the interacting modes. In our framework, we use modes of the same symmetry, thus with an inherently good spatial overlap, and a high- $Q$ factor to enhance FWM efficiency. We study the case of degenerate FWM (dFWM), where the two pump photons are at equal energy. In this process, we have two input frequencies $\omega_{1}$ and $\omega_{2}$, that are commonly referred to as the pump and idler. The frequency of the signal photon generated via dFWM we denote as $\omega_{3}=2 \omega_{1}+\omega_{2}$. To reproduce dFWM in our numerical simulations, we define the nonlinear polarization at $\omega_{3}$, as $\mathbf{P}^{\omega_{3}}=$ $3 \varepsilon_{0} \chi^{(3)}\left[2\left(\mathbf{E}^{\omega_{1}} \cdot \mathbf{E}^{\omega_{2}}\right) \mathbf{E}^{\omega_{1}}+\left(\mathbf{E}^{\omega_{1}} \cdot \mathbf{E}^{\omega_{1}}\right) \mathbf{E}^{\omega_{2}}\right]$, where $\mathbf{E}^{\omega_{1}}$ and $\mathbf{E}^{\omega_{2}}$ are the electric field at frequency $\omega_{1}$ and $\omega_{2}$, respectively. In our calculations, the pump and idler have the same optical power $P_{0}$, and thus the dFWM conversion efficiency is $\gamma=$ $P_{\omega_{3}} / P_{0}^{3}$. Figure $4($ a) shows the dFWM efficiency as a function of the idler wavelength in the vicinity of the MQ resonance when the frequency of the pump is fixed at the quasi-BIC. Figures 4(b) and 4(c) show the dFWM efficiency as a function of the idler wavelength in the vicinity of MD resonance when the frequency of the pump is fixed at the quasi-BIC and MQ mode, respectively. It follows from Fig. 4(a) that, when we use the quasi-BIC as pump and the MQ resonance as the idler, near-IR photon generation with wavelength of $1240 \mathrm{~nm}$ via dFWM can achieve a peak efficiency of $4 \times 10^{-5}$. From these results, it is clear that the $Q$ factor of the pump affects strongly the dFWM efficiency. Indeed, for the same idler

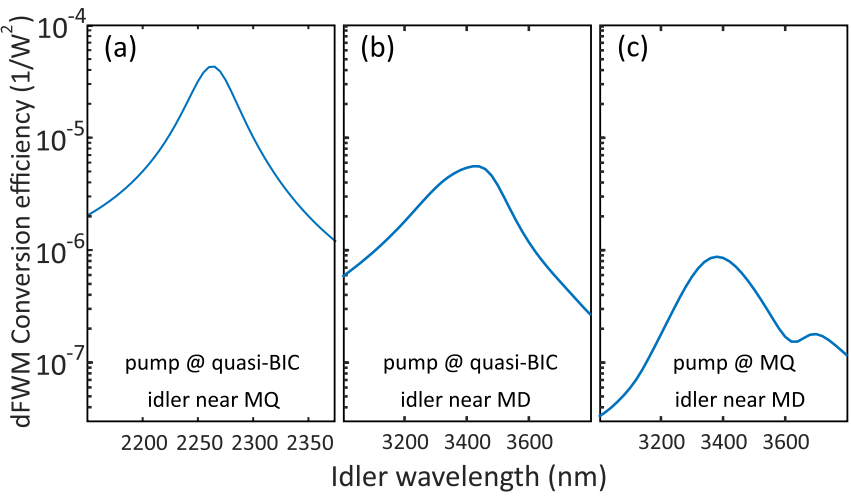

FIG. 4. Comparison of different parametric processes. (a) Conversion efficiency of dFWM as a function of the idler wavelength in the vicinity of the MQ resonance, when the pump wavelength is fixed at the quasi-BIC. (b), (c) Same as (a) but for the idler wavelength in the vicinity of the MD resonance, when the pump wavelength is fixed at (b) quasi-BIC and (c) MQ mode. 

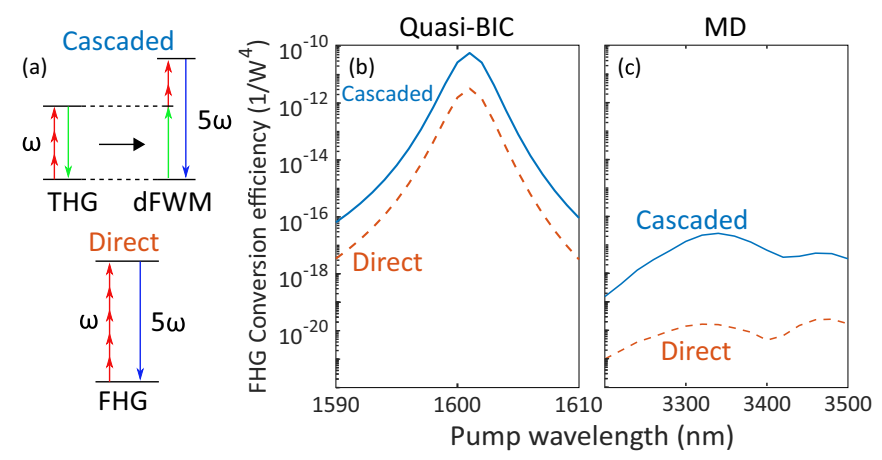

FIG. 5. Fifth-harmonic generation. (a) Schemes of cascaded and direct FHG. (b), (c) Conversion efficiency of FHG as a function of the pump wavelength in the vicinity of quasi-BIC (b) and MD mode (c). Direct (dashed line) and cascaded (solid line) processes are shown.

mode, the quasi-BIC driven $\mathrm{dFWM}$ is about 10 times more efficient with respect to the MQ-driven dFWM [see Figs. 4(b) and 4(c)]. This can be qualitatively explained by considering that, neglecting spectral and spatial overlap between the pump and idler modes, the dFWM efficiency scales as the product $Q_{1}^{2} \times Q_{2}$ where $Q_{1}$ and $Q_{2}$ are the $Q$ factors of the modes at $\omega_{1}$ and $\omega_{2}[41]$.

\section{Fifth-harmonic generation}

As the most intriguing result, here we consider the potential of quasi-BIC for fifth-harmonic generation (FHG) at the nanoscale. High-harmonics generation is well studied in gases (see, e.g., Refs. [42,43]) and less in solid state physics. It was shown recently that nanostructuring, precise chemical engineering, and tailoring of the optical state density give unique possibilities to control high-harmonic generation in nanostructures. Previous work mainly analyzed high-order harmonic generation in bulk materials, thin layers, and metasurfaces [44-47]. Here we show that FHG can be very efficient in a single silicon nanodisk supporting quasi-BIC. We consider two of the possible processes generating fifth harmonic. The first is via the fifth-order nonlinear susceptibility and the second is via a cascaded $\chi^{(3)}$ effect where THG is followed by dFWM between the pump and the THG signal. The schemes of these processes are shown in Fig. 5(a). The cascaded and direct FHG in centrosymmetric crystals was first observed in $\mathrm{CaCO}_{3}$ [48] and has received later on a great deal of attention. The advantages of the cascaded process are its higher efficiency and extremely short time constant $[35,36]$. In our case, we perform numerical simulations of the direct FHG defining the nonlinear polarization as $\mathbf{P}^{5 \omega}=$ $3 \varepsilon_{0} \chi^{(5)}(\mathbf{E} \cdot \mathbf{E})^{2} \mathbf{E}^{\omega}$ where $\chi^{(5)}$ is the fifth-order susceptibility. We estimated $\chi^{(5)}$ based on the phenomenological relation $\chi^{(n)} \approx \chi(1) / E_{a t}^{(n-1)}$, where $E_{a t}$ is the characteristic atomic field strength, that applied to the fifth order in Si gives $\chi^{(5)} \approx$ $5.6 \times 10^{-39}(\mathrm{~m} / \mathrm{V})^{4}$ [1]. With this approach, we obtained the FHG conversion efficiency spectra from quasi-BIC and MD modes reported in Figs. 5(b) and 5(c), respectively. As we can observe, the highest conversion efficiency is achieved exploiting the quasi-BIC. The cascaded pathway for FHG that we consider relies on the third-order nonlinear susceptibility which is larger than $\chi^{(5)}$. It is thus interesting to evaluate the relative efficiency between the direct and cascading effects. For the latter, we first evaluated THG from the pump photons and then evaluated dFWM where the idler is the THG light. The solid lines in Fig. 5 show the conversion efficiencies obtained for the cascaded effect. Interestingly, the efficiency is higher in this case compared to the one obtained from the direct effect. This is due to the relation between the $\chi^{(5)}$ and $\chi^{(3)}$ that is given by $\chi^{(5)} \approx\left[\chi^{(3)}\right]^{2} / \chi^{(1)}$.

\section{CONCLUSIONS}

We have suggested an efficient way to realize multifrequency and cascaded parametric nonlinear processes at the nanoscale. We have combined high- $Q$ quasinormal modes of nanoscale resonators originating from the BIC concept with a nontrivial engineering of the substrate, and we have predicted a boost of the efficiency of nonlinear effects at the nanoscale at least by two orders of magnitude. In particular, we have reported examples related to THG, dFWM, and cascaded harmonic generation, expanding substantially the range of applications of resonant nonlinear nanophotonics. Subwavelength structures enable the development of highperformance nanoscale devices for many applications such as high-harmonic generation, quantum imaging, and broadband spectroscopy at a few photon level $[25,49,50]$. In this context, strong nonlinear effects at the nanoscale are expected to enhance the performance of the next-generation integrated photonic devices and resolve many challenges encountered in the transition from concept demonstration to viable technology.

\section{ACKNOWLEDGMENTS}

S.K., A.B., and Y.K. acknowledge useful collaboration and discussions with K. Koshelev. L.C. acknowledges STAR$\mathrm{StG}$ project $\mathrm{PULSAR}$. C.D.A. acknowledges financial support from Consiglio Nazionale delle Ricerche (CNR) joint laboratory projects 2019-2021. A.A.B. acknowledges RSF (Grant No. 18-72-10140) and Foundation for the Advancement of Theoretical Physics and Mathematics BASIS. This work was supported by the Strategic Fund of the Australian National University. A.B. acknowledges support by the RFBR (19-0200419), the Grant of the President of the Russian Federation (MK-403.2018.2), and "BASIS" foundation.
[1] R. Boyd, Nonlinear Optics (Academic, Burlington, MA, 2003).

[2] A. Rodriguez, M. Soljačić, J. D. Joannopoulos, and S. G. Johnson, $\chi^{(2)}$ and $\chi^{(3)}$ harmonic generation at a critical power in inhomogeneous doubly resonant cavities, Opt. Express 15, 7303 (2007).

[3] J. I. Dadap, J. Shan, and T. F. Heinz, Theory of optical second-harmonic generation from a sphere of centrosymmetric 
material: small-particle limit, J. Opt. Soc. Am. B 21, 1328 (2004).

[4] M. Finazzi, P. Biagioni, M. Celebrano, and L. Duo, Selection rules for second-harmonic generation in nanoparticles, Phys. Rev. B 76, 125414 (2007).

[5] K. S. Frizyuk, Second-harmonic generation in dielectric nanoparticles with different symmetries, J. Opt. Soc. Am. B 36, F32 (2019).

[6] M. Kauranen and A. V. Zayats, Nonlinear plasmonics, Nat. Photonics 6, 737 (2012).

[7] H. Wu, S. Pan, K. R. Poeppelmeier, H. Li, D. Jia, Z. Chen, X. Fan, Y. Yang, J. M. Rondinelli, and H. Luo, $\mathrm{K}_{3} \mathrm{~B}_{6} \mathrm{O}_{10} \mathrm{Cl}$ : a new structure analogous to perovskite with a large second harmonic generation response and deep UV absorption edge, J. Am. Chem. Soc. 133, 7786 (2011).

[8] S. Y. Hong, J. I. Dadap, N. Petrone, P. C. Yeh, J. Hone, and R. M. Osgood Jr., Optical Third-Harmonic Generation in Graphene, Phys. Rev. X 3, 021014 (2013).

[9] A. Krasnok, M. Tymchenko, and A. Alu, Nonlinear metasurfaces: a paradigm shift in nonlinear optics, Mater. Today 21, 8 (2018).

[10] C. Renaut, L. Lang, K. Frizyuk, M. Timofeeva, F. Komissarenko, I. Mukhin, D. Smirnova, F. Timpu, M. Petrov, Y. Kivshar, and R. Grange, Reshaping the second-order polar response of hybrid metal-dielectric nanodimers, Nano Lett. 19, 877 (2019).

[11] A. Kuznetsov, A. E. Miroshichenko, M. I. Brongersma, Y. S. Kivshar, and B. Lukyanchuk, Optically resonant dielectric nanostructures, Science 354, aag2472 (2016).

[12] S. Kruk and Y. Kivshar, Functional meta-optics and nanophotonics governed by Mie resonances, ACS Photonics 4, 2638 (2017).

[13] Y. S. Kivshar, All-dielectric meta-optics and nonlinear nanophotonics, Natl. Sci. Rev. 5, 144 (2018).

[14] M. R. Shcherbakov, D. N. Neshev, B. Hopkins, A. S. Shorokhov, I. Staude, E. V. Melik-Gaykazyan, M. Decker, A. A. Ezhov, A. E. Miroshnichenko, I. Brener, A. Fedyanin, and Y. Kivshar, Enhanced third-harmonic generation in silicon nanoparticles driven by magnetic response, Nano Lett. 14, 6488 (2014).

[15] L. Xu, M. Rahmani, K. Z. Kamali, A. Lamprianidis, L. Ghirardini, J. Sautter, R. Camacho-Morales, H. Chen, M. Parry, I. Staude, G. Zhang, D. Neshev, and A. E. Miroshnichenko, Boosting third-harmonic generation by a mirror-enhanced anapole resonator, Light: Sci. Appl. 7, 44 (2018).

[16] G. Grinblat, Yi Li, M. P. Nielsen, R. F. Oulton, and S. Maeir, Degenerate four-wave mixing in a multiresonat germanium naodisk, ACS Photonics 4, 2144 (2017).

[17] R. Colom, L. Xu, L. Marini, F. Bedu, I. Ozerov, T. Begou, J. Lumeau, A. E. Miroshnishenko, D. Neshev, B. T. Kuhlmey, S. Palomba, and N. Bonod, Enhanced four-wave mixing in doubly resonant Si nanoresonators, ACS Photonics 6, 1295 (2019).

[18] L. Carletti, A. Locatelli, O. Stepanenko, G. Leo, and C. De Angelis, Enhanced second-harmonic generation from magnetic resonance in AlGaAs nanoantennas, Opt. Express 23, 26544 (2015).

[19] V. F. Gili, L. Carletti, A. Locatelli, D. Rocco, M. Finazzi, L. Ghirardini, I. Favero, C. Gomez, A. Lemaitre, M. Celebrano, C. De Angelis, and G. Leo, Monolithic AlGaAs second-harmonic nanoantennas, Opt. Express 24, 15965 (2016).
[20] R. Camacho Morales, M. Rahmani, S. Kruk, L. Wang, L. Xu, D. A. Smirnova, A. S. Solntsev, A. Miroshnichenko, H. H. Tan, F. Karouta, S. Naureen, K. Vora, L. Carletti, C. De Angelis, C. Jagadish, Y. S. Kivshar, and D. N. Neshev, Nonlinear generation of vector beams from AlGaAs nanoantennas, Nano Lett. 16, 7191 (2016).

[21] S. Kruk, R. Camacho-Morales, L. Xu, M. Rahmani, D. A. Smirnova, L. Wang, H.-H. Tan, C. Jagadish, D. N. Neshev, and Y. S. Kivshar, Nonlinear optical magnetism revealed by second-harmonic generation in nanoantennas, Nano Lett. 17, 3914 (2017).

[22] F. Timpu, A. Sergeyev, N. R. Hendricks, and R. Grange, Second-harmonic enhancement with Mie resonances in perovskite nanoparticles, ACS Photonics 4, 76 (2017).

[23] S. Liu, P. P. Vabishchevich, A. Vaskin, J. L. Reno, G. A. Keeler, M. B. Sinclair, I. Staude, and I. Brener, An all-dielectric metasurface as a broadband optical frequency mixer, Nat. Commun. 9, 2507 (2018).

[24] F. Timpu, J. Sendra, C. Renaut, L. Lang, M. Timofeeva, M. T. Buscaglia, V. Buscaglia, and R. Grange, Lithium niobate nanocubes as linear and nonlinear ultraviolet Mie resonators, ACS Photonics 6, 545 (2019).

[25] H. Liu, C. Guo, G. Vampa, J. L. Zhang, T. Sarmiento, M. Xiao, P. H. Bucksbaum, J. Vuĉović, S. Fan, and D. A. Reis, Enhanced high-harmonic generation from an all-dielectric metasurface, Nat. Phys. 14, 1006 (2018).

[26] B. Sain, C. Meier, and T. Zentgraf, Nonlinear optics in all-dielectric nanoantennas and metasurfaces: a review, Adv. Photonics 1, 024002 (2019).

[27] M. V. Rybin, K. L. Koshelev, Z. F. Sadrieva, K. B. Samusev, A. A. Bogdanov, M. F. Limonov, and Y. S. Kivshar, High$Q$ Supercavity Modes in Subwalength Dielectric Resonators, Phys. Rev. Lett. 119, 243901 (2017).

[28] A. A. Bogdanov, K. L. Koshelev, P. V. Kapitanova, M. V. Rybin, S. A. Gladyshev, Z. F. Sadrieva, K. B. Samusev, Y. S. Kivshar, and M. F. Limonov, Bound states in the continuum and Fano resonances in the strong mode coupling regime, Adv. Photonics 1, 016001 (2019).

[29] C. W. Hsu, Bo Zhen, A. D. Stone, J. D. Joannopoulos, and M. Soljačić, Bound states in the continuum, Nat. Rev. Mater. 1, 16048 (2016).

[30] L. Carletti, K. Koshelev, C. De Angelis, and Y. S. Kivshar, Giant Nonlinear Response at the Nanoscale Driven by Bound States in the Continuum, Phys. Rev. Lett. 121, 033903 (2018).

[31] E. V. Melik-Gaykazyan, K. L. Koshelev, J.-H. Choi, S. S. Kruk, H.-G. Park, A. A. Fedyanin, and Y. S. Kivshar, Enhanced second-harmonic generation with structured light in AlGaAs nanoparticles governed by magnetic response, JETP Lett. 109, 131 (2019).

[32] K. Koshelev, A. Bogdanov, and Y. S. Kivshar, Meta-optics and bound states in the continuum, Sci. Bull. 64, 836 (2019).

[33] N. Z. Alam, S. A. Schultz, J. Upham, I. De Leon, and R. W. Boyd, Large optical nonlinearuty of nanoantennas coupled to an epsilon-near-zero material, Nat. Photonics 12, 79 (2018).

[34] D. Rocco, M. A. Vincenti, and C. De Angelis, Boosting second harmonic radiation from AlGaAs nanoantennas with epsilonnear-zero materials, Appl. Sci. 8, 2212 (2018). 
[35] S. Akhmanov, A. Dubovik, S. Saltiel, I. Tomov, and V. Tunkin, Nonlinear optical effects of the fourth order along the field in lithium formiate crystal, Pis'ma Zh. Eksp. Teor. Fiz. 20, 264 (1974) [JETP Lett. 20, 117 (1974)].

[36] S. M. Saltiel, A. A. Sukhorukov, and Y. S. Kivshar, Multistep parametric processes in nonlinear optics, Prog. Opt. 47, 1 (2005).

[37] M. A. Green, Self-consistent optical parameters of intrinsic silicon at $300 \mathrm{~K}$ including temperature coefficients, Sol. Energ. Mat. Sol. Cells 92, 1305 (2008).

[38] H. Friedrich and D. Wintgen, Interfering resonances and bound states in the continuum, Phys. Rev. A 32, 3231 (1985).

[39] A. E. Miroshnichenko, A. B. Evlyukhin, Y. S. Kivshar, and B. N. Chichkov, Substrate-induced resonant magnetoelectric effects for dielectric nanoparticles, ACS Photonics 2, 1423 (2015).

[40] I. Sinev, I. Iorsh, A. Bogdanov, D. Permyakov, F. Komissarenko, I. Mukhin, A. Samusev, V. Valuckas, A. Kuznetsov, B. Luk'yanchuk, A. Miroshnichenko, and Y. S. Kivshar, Polarization control over electric and magnetic dipole resonances of dielectric nanoparticles on metallic films, Laser Photonics Rev. 10, 799 (2016).

[41] Z. Lin, X. Liang, M. Lončar, S. G. Johnson, and A. W. Rodriguez, Cavity-enhanced second-harmonic generation via nonlinear-overlap optimization, Optica 3, 233 (2016).

[42] P. B. Corkum, Plasma Perspective on Strong Field Multiphoton Ionization, Phys. Rev. Lett. 71, 1994 (1993).
[43] A. L'Huillier and P. Balcou, High-Order Harmonic Generation in Rare Gases with a 1-ps 1053-nm Laser, Phys. Rev. Lett. 70, 774 (1993).

[44] M. Sivis, M. Taucer, G. Vampa, K. Johnston, A. Staudte, A. Y Naumov, D. M. Villeneuve, C. Ropers, and P. B. Corkum, Tailored semiconductors for high-harmonic optoelectronics, Science 357, 303 (2017).

[45] G. Vampa, B. G. Ghamsari, S. Siadat Mousavi, T. J. Hammond, A. Olivieri, E. Lisicka-Skrek, A. Yu Naumov, D. M. Villeneuve, A. Staudte, P. Berini, and P. B. Corkum, Plasmon-enhanced high-harmonic generation from silicon, Nat. Phys. 13, 659 (2017).

[46] S. Kim, J. Jin, Y.-J. Kim, I.-Y. Park, Y. Kim, and S.-W. Kim, High-harmonic generation by resonant plasmon field enhancement, Nature (London) 453, 757 (2008).

[47] Y. Gao, H. Lee, J. Jiao, B. J. Chun, S. Kim, D.-H. Kim, and Y.-J. Kim, Surface third and fifth harmonic generation at crystalline $\mathrm{Si}$ for non-invasive inspection of Si wafers inter-layer defects, Opt. Express 26, 32812 (2018).

[48] S. Akhmanov, V. Martinov, S. Saltiel, and V. Tunkin, Nonresonant six photon process in calcite crystal, Pis'ma Zh. Eksp. Teor. Fiz. 22, 143 (1975) [JETP Lett. 22, 65 (1975)].

[49] G. B. Lemos, V. Borish, G. D. Cole, S. Ramelow, R. Lapkiewicz, and A. Zeilinger, Quantum imaging with undetected photons, Nature (London) 512, 409 (2014).

[50] P. Cheben, R. Halir, J. H. Schmid, H. A. Atwater, and D. R. Smith, Subwavelength intergrated photoncs, Nature (London) 560, 565 (2018). 